\title{
Direct Observation of Sub-Poissonian Number Statistics in a Degenerate Bose Gas
}

\author{
C.-S. Chuu, F. Schreck团 T. P. Meyrath, J. L. Hanssen It G. N. Price, and M. G. Raizen \\ Center for Nonlinear Dynamics and Department of Physics, \\ The University of Texas at Austin, Austin, TX 78712, USA
}

(Dated: December 1, 2018)

\begin{abstract}
We report the direct observation of sub-Poissonian number fluctuation for a degenerate Bose gas confined in an optical trap. Reduction of number fluctuations below the Poissonian limit is observed for average numbers that range from 300 to 60 atoms.

PACS numbers: 03.75.Hh, 32.80.Pj
\end{abstract}

The study of the quantum statistics of light has been at the heart of modern quantum optics for many years, with examples ranging from photon anti-bunching [1] and squeezed states of light 2], to quantum communication 3]. The emerging field of atom optics has now reached the stage where the direct measurement of atom statistics can have a similar impact. In particular, novel quantum statistics have been predicted for quantum degenerate gases under a wide range of conditions. Two recent examples are the prediction of sub-Poissonian statistics and Fock state production in the Mott insulator transition [4] and in the Quantum Tweezer [5], and atomic antibunching in a one-dimensional gas of repulsive bosons (Tonks-Girardeau gas) [6]. More generally, it is clear that the controlled study of entanglement and quantum computing with massive particles must be based on the detection at single-atom or ion level.

Following this theoretical work, an early experiment reported number squeezing in an optical lattice based on the observation of increased phase noise [7]. Subsequent experiments have provided clear and convincing evidence of the Mott insulator state [8]. In parallel work, several groups have observed novel behavior of a 1-D gas in the Tonks regime [9]. However all of these experiments were conducted with a large number of atoms and were therefore not statistical in nature.

In order to directly probe the atom statistics of these novel states, one must incorporate single-atom counting with a Bose-Einstein condensate (BEC) apparatus. We report in this Letter the experimental realization of such a system and the first direct measurement of subPoissonian atom number statistics in a degenerate Bose gas.

The fluctuations of atom number within a small volume in a classical ideal gas is given by $\sigma_{N}=N^{1 / 2}$, where $N$ is the mean atom number [10]. For the fluctuations of atom number in a degenerate Bose gas this is not necessarily true and has been the topic of intense theoretical debate. In the case of an ideal Bose gas, number fluctua-

\footnotetext{
*Present address: Institut für Quantenoptik und Quanteninformation, Innsbruck, Austria.

${ }^{\dagger}$ Present address: National Institute of Standards and Technology, Gaithersburg, MD 20899, USA.
}

tions have been studied in a box [11] and, more recently, in a harmonic trap 12, 13. for microcanonical and canonical ensembles. For a weakly interacting Bose gas, number fluctuations were first investigated in Ref. 14]. The role of interactions was then further studied by including the effect of the thermal excitation of phonons in the thermodynamic limit with number-nonconserving [15] and number-conserving [16] Bogoliubov methods. Most recently, an isolated system of finite atom number was considered for studying number fluctuations in a harmonic trap [17] and in a box [18]. The result obtained in the latter case, in particular, predicted number fluctuation proportional to $N^{1 / 2}$.

In BEC experiments reported thus far, typical shot-toshot number fluctuations greatly exceed the Poissonian limit, presumably due to technical noise. Here we report on an ultra-stable optical trap which has a controllable trapping volume and depth. This trap can be used to achieve sub-Poissonian number fluctuations by the following mechanism: for a fully loaded trap, the potential depth $U_{0}$ in the shallowest direction is equal to the chemical potential $\mu$ of the degenerate Bose gas if one neglects tunnelling out of the trap. In the Thomas-Fermi (TF) limit, the atom number $N$ is proportional to $\mu^{5 / 2}$ for a harmonic trapping potential. The atom number is thus related to the trap depth as $N \propto U_{0}^{5 / 2}$. From this, it is clear that a precise control of the trap depth can lead to a precise control of the atom number. This conclusion remains valid even when assuming tunnelling and a realistic nonharmonic potential.

Our experimental apparatus for studying subPoissonian number statistics is similar to our previous work [19]. A BEC of $2 \times 10^{5}{ }^{87} \mathrm{Rb}$ atoms is produced by evaporation in a large volume optical dipole trap. The BEC is then compressed and transferred to the final small-volume optical trap. This trap is formed by five Gaussian sheets, with two pairs propagating vertically and one horizontal sheet to hold the atoms against gravity, shown pictorially in Fig. 1(a). The calculated potentials given by the measured beam parameters are shown in Fig. 1(b) to Fig. 1(d) for the respective directions. All beams originated from a $10 \mathrm{~W}$ laser at $\lambda=532 \mathrm{~nm}$ are tightly focused in one axis at the position of the condensate. Each sheet pair is derived from the first order deflections of multiple frequency acousto-optical modu- 

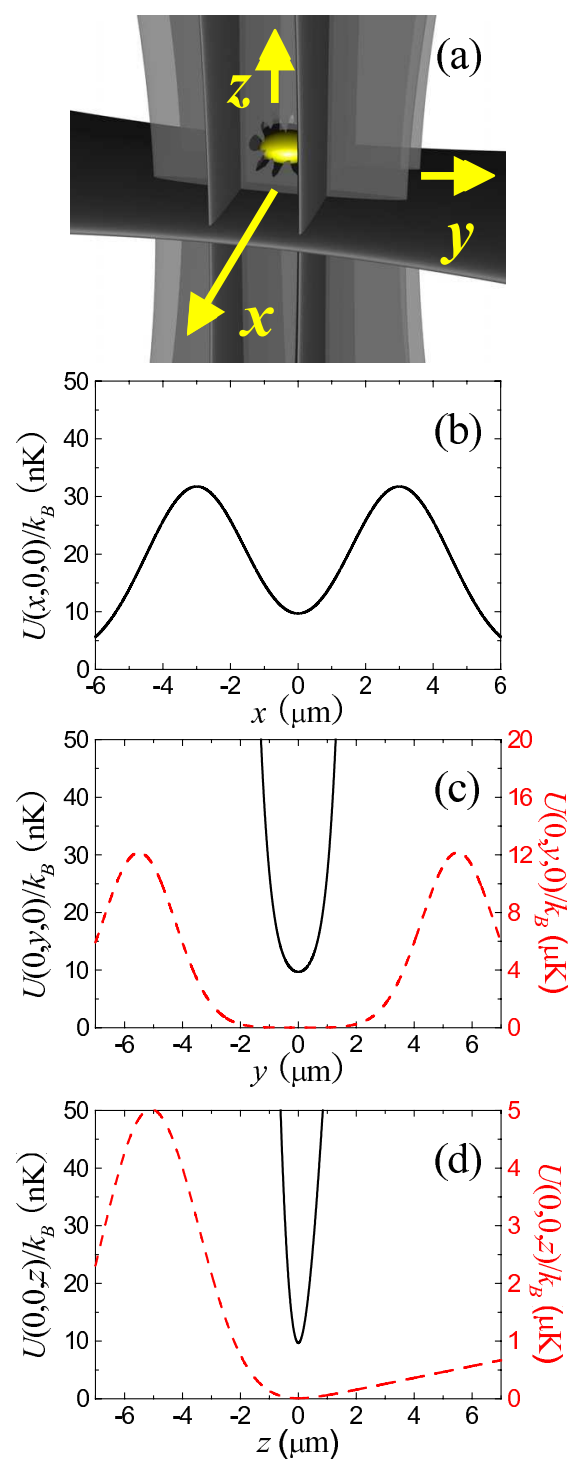

FIG. 1: (color online) The optical trap. (a) Beam pictorial. Gravity points in $-z$ direction in this pictorial. (b) This plot shows the potential energy $U$ at $y=z=0$. In the $x$ direction, the atoms are confined by two Gaussian sheets with a separation of $l_{x}=5 \mu \mathrm{m}$. (c) Confinement in the $y$ direction is of similar shape to the $x$ direction but deeper. (d) In the $z$ direction, gravity and a Gaussian sheet form a gravito-optical trap. The dashed curves in (c) and (d) show the same potentials for the different scales given on the right axes. Each potential plot is calculated based on measured beam characteristics and are appropriate for order 100 atoms.

lators, providing independent control of the position and power [20]. The sheet pairs and the horizontal sheet have a maximum power of $P_{x}^{\max }=25 \mathrm{~mW}, P_{y}^{\max }=80 \mathrm{~mW}$, $P_{z}^{\max }=100 \mathrm{~mW}$ per sheet and a $1 / \mathrm{e}^{2}$ radius of $2.5 \mu \mathrm{m}$ $\times 100 \mu \mathrm{m}$ for the $x$ and $y$ axes and $3.4 \mu \mathrm{m} \times 200 \mu \mathrm{m}$ for the $z$ axis, respectively; $x, y$ and $z$ refer to the potential axes of Fig. 1] For typical operating conditions, the trap has a depth of $U_{0} / k_{B}=22 \mathrm{nK}$ (for $P_{x}=0.2 \mathrm{~mW}$ )

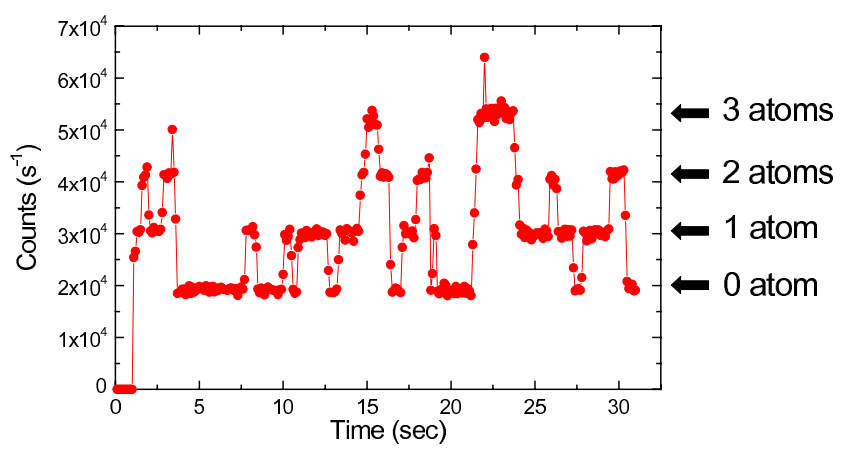

FIG. 2: (color online) Step-wise signal of the APD. The atom number from CCD fluorescence imaging is calibrated by an APD operating in photon counting mode. The fluorescence counting rate per atom is $10^{4} \mathrm{~s}^{-1}$ with a background of $2 \times$ $10^{4} \mathrm{~s}^{-1}$. The signals shown are for random loading of the MOT from background vapor. The time step of fluorescence binning is $100 \mathrm{~ms}$.

with the weakest trapping potential in the $x$ direction and a geometric mean trapping frequency of $\bar{\omega}=2 \pi \times$ $300 \mathrm{~Hz} ; k_{B}$ is Boltzmann's constant. In the final evaporation stage $U_{0}$ is ramped down adiabatically over a period of $1500 \mathrm{~ms}$ with an exponential shape. $U_{0}$, being the lowest evaporation barrier, determines the chemical potential and thus the atom number. Its final value is varied to obtain different atom numbers.

Two methods are employed for measurements of atom numbers. For numbers of order $10^{3}$ or larger, absorption imaging is used yielding spatial and number information. At lower atom numbers however, fluorescence imaging is used because of higher signal-to-noise ratio in this regime. This is accomplished by transferring the atoms into a small magneto-optical trap (MOT) [21]. The MOT uses six beams with a diameter of $1 \mathrm{~mm}$, an intensity of $65 \mathrm{~mW} / \mathrm{cm}^{2}$, a detuning of about $10 \mathrm{MHz}$, and a magnetic field gradient of $260 \mathrm{G} / \mathrm{cm}$. Transfer from the optical trap to the MOT shows a saturation behavior with MOT beam intensity, indicating that all atoms are captured. The resulting fluorescence signal is detected by a charge-coupled-device (CCD) camera for $100 \mathrm{~ms}$ and is calibrated against an avalanche photodiode (APD). Because of the low density during exposure, there is little possibility for multiple scattering events during detection. Therefore, the measured fluorescence signal from the MOT is proportional to the number of atoms present.

The calibration of the atom number obtained from fluorescence imaging is accomplished by operating the MOT in a regime 22] where discrete fluorescence levels of different small atom numbers are observable on an APD, as shown in Fig. 2] The MOT is then suddenly switched to the typical operating settings described above and the new fluorescence level is obtained for the same atom number. This yields an absolute accuracy in atom number better than $\pm 10 \%$ 23]. The result is consistent with calculations of the ratio of scattering rates for the given settings [24]. Atom numbers may also be estimated by 

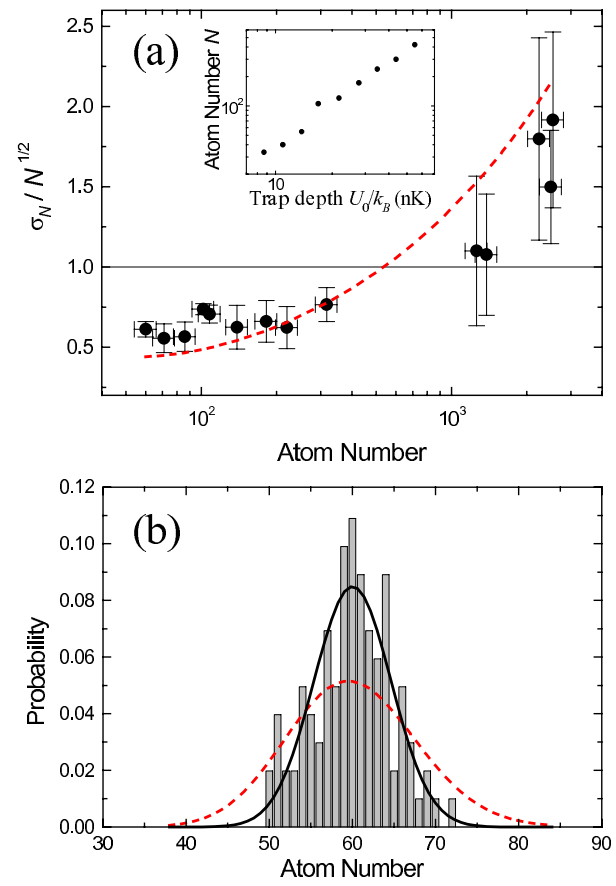

FIG. 3: (color online) Observation of sub-Poissonian number statistics. (a) The solid circles show the measured fluctuation normalized to the Poissonian case as a function of atom number. The vertical error bars are the $68 \%$ confidence intervals for each measurement. The horizontal error bars represent the absolute accuracy in atom number. Sub-Poissonian number statistics is observed for atom numbers below 500 where the fluctuations due to technical noise are not larger than the Poissonian fluctuation. The dashed curve is the estimated fluctuation from technical noise and background capture. The inset shows the measured atom number as a function of the trap depth $U_{0}$. (b) Histogram of 100 sample points shows sub-Poissonian statistics for mean atom number $N=60$. The solid curve is the Gaussian fit to the distribution. The dashed curve is the Poissonian distribution with the same mean atom number.

using the TF approximation with $\mu=U_{0}$ and modelling the trap by a harmonic potential. This yields a $35 \%$ deviation below the measured atom numbers, indicating rough accuracy of this model.

Due to inhomogeneity of the optical potential, after the potential barrier is lowered, some atoms remain outside of the trap. These atoms are removed before the final number detection by raising the potential barrier to its maximum intensity $\left(U_{0}^{\max } / k_{B}=3 \mu \mathrm{K}\right)$ and using a supplementary optical sheet pair to sweep the residual atoms away from the well. A magnetic gradient is also applied to remove atoms outside the range of the sweeping beams.

Fig. 31(a) shows the measured fluctuations normalized to the Poissonian case $\sigma_{N} / N^{1 / 2}$ (solid circles) as a function of atom number. Sub-Poissonian fluctuations are observed for atom numbers below 500, where the technical noise is no longer dominant 25]. The measured fluc- tuation at $N=60$ atoms is approximately $60 \%$ of the corresponding Poissonian fluctuation. This series of 100 measurements is shown as a histogram plot in Fig. 3(b). A Gaussian fit to the data indicates a measured standard deviation of $\sigma_{N} / N=7.9 \%$ with a $99 \%$ confidence interval of $[7.4 \%, 8.5 \%]$ 26]. This indicates a reliable measurement of deviation well below the Poissonian value of $N^{1 / 2} / N=(12.9 \pm 0.5) \%$, where the error is given by absolute accuracy in atom number. Several possible technical noise sources were measured and estimates of their contribution to the atom number fluctuation result in the following [25]: $2.0 \%$ from $P_{x}, 2.4 \%$ from $P_{y}, 0.1 \%$ from $P_{z}, 2.2 \%$ from $l_{x}$, and $2.0 \%$ from $l_{y}$. The overall contribution due to technical noise gives an expected atom number fluctuation of $\delta_{\text {tech }}=4.3 \%$, which is very close to what is measured for larger atom numbers. For lower atom numbers, background capture during the detection is a major contribution to the measured fluctuation. The background capture during the $100 \mathrm{~ms}$ image-taking process with no atoms present is measured to have a mean of $N_{\text {bg }}=5$ atoms. This random process, which has Poissonian statistics, broadens the measured atom number distribution. A simple estimate of atom number fluctuation from both technical noise and background capture, assuming a constant background capture for different atom number $N$, results in $\sqrt{\left(\delta_{\text {tech }}\right)^{2}+\left(\left(N_{\mathrm{bg}}\right)^{1 / 2} / N\right)^{2}}$, shown as the dashed curve in Fig. B(a). This result gives a similar increasing tendency as for the measured fluctuations. A more detailed calculation without TF approximation shows an increase in sensitivity to trap fluctuations at lower atom numbers $[27]$.

We have measured the dependence of atom statistics on the ramp time, $t_{\mathrm{ramp}}$, in order to probe the manybody dynamics. The results are displayed in Fig. 4 where the fluctuations (normalized to the Poissonian case) are plotted as a function of $t_{\mathrm{ramp}}$. We find that for time scales shorter than $250 \mathrm{~ms}$, the atom statistics become super-Poissonian, while for longer times they are subPoissonian. This result provides the timescale for adiabatic following, a key feature of the process. The theoretical analysis of our system is yet to be completed, and requires the development of a time-dependent many-body theory without a mean-field approximation. Surveying previous theoretical work, the closest case we have found is the analysis of relative number fluctuations between two condensates separated by a tunnel barrier that is ramped up in time [28]. The authors of that paper found sub-Poissonian fluctuations in the relative atom number under appropriate conditions. However our system is considerably different, with a single trap in a quantum degenerate regime undergoing loss of atoms as the barrier is lowered. Recent work on quantum kinetic theory may provide insights to the present system [29] and finite-size trap effects are also being considered [30].

In conclusion, we have observed sub-Poissonian number statistics in a degenerate Bose gas prepared in an optical dipole trap. By precisely controlling the chemical potential, we obtained a wide range of atom num- 


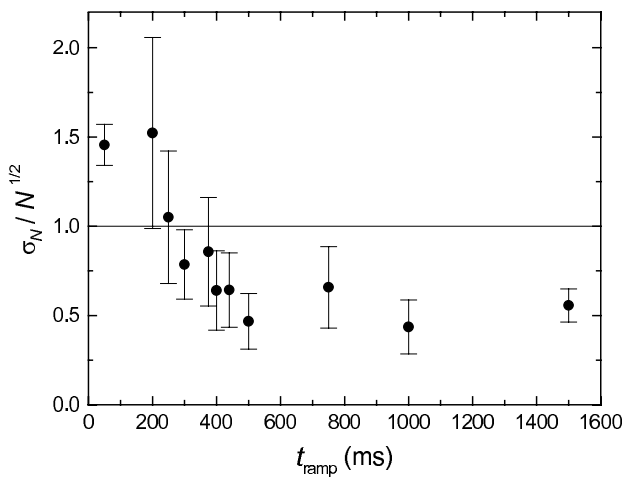

FIG. 4: Atom number fluctuations (normalized to the Poissonian case) as a function of ramp time. Adiabaticity breaks down when the ramp time is shorter than $250 \mathrm{~ms}$. The error bars show the $68 \%$ confidence intervals for each measurement. The horizontal line shows the level of Poissonian fluctuation. bers starting at several tens and going up to a few thousand atoms. For atom numbers below a few hundred, we observed sub-Poissonian number statistics. Future work will be to eliminate sources of technical noise and to approach the $N=1$ Fock state.

The authors would like to acknowledge support from the Sid W. Richardson Foundation, the National Science Foundation, the R. A. Welch Foundation, and the Alexander von Humboldt Foundation. We also thank A. M. Dudarev and Q. Niu for useful discussions.
[1] H. J. Kimble, M. Dagenais, and L. Mandel, Phys. Rev. Lett. 39, 691 (1977).

[2] R. E. Slusher, L. W. Hollberg, B. Yurke, J. C. Mertz, and J. F. Valley, Phys. Rev. Lett. 55, 2409 (1985); L.-A. Wu, H. J. Kimble, J. L. Hall, and H. Wu, Phys. Rev. Lett. 57, 2520 (1986); M. G. Raizen, L. A. Orozco, M. Xiao, T. L. Boyd, and H. J. Kimble, Phys. Rev. Lett. 59, 198 (1987); Y. Yamamoto and H. A. Haus, Rev. Mod. Phys. 58, 1001 (1986).

[3] S. L. Braunstein and P. van Loock, Rev. Mod. Phys. 77, 513 (2005).

[4] D. Jaksch, C. Bruder, J. I. Cirac, C. W. Gardiner, and P. Zoller, Phys. Rev. Lett. 81, 3108 (1998).

[5] R. B. Diener, B. Wu, M. G. Raizen, and Q. Niu, Phys. Rev. Lett. 89, 070401 (2002).

[6] K. V. Kheruntsyan, D. M. Gangardt, P. D. Drummond, and G. V. Shlyapnikov, Phys. Rev. Lett. 91, 040403 (2003).

[7] C. Orzel, A. K. Tuchman, M. L. Fenselau, M. Yasuda, and M. A. Kasevich, Science 291, 2386 (2001).

[8] M. Greiner, O. Mandel, T. Esslinger, T. W. Hänsch, and I. Bloch, Nature 415, 39 (2002); M. Greiner, O. Mandel, T. W. Hänsch, and I. Bloch, Nature 419, 51 (2002); F. Gerbier, A. Widera, S. Fölling, O. Mandel, T. Gericke, and I. Bloch, Phys. Rev. Lett. 95, 050404 (2005).

[9] T. Kinoshita, T. Wenger, and D. S. Weiss, Science 305, 1125 (2004); B. Paredes, A. Widera, V. Murg, O. Mandel, S. Fölling, I. Cirac, G. V. Shlyapnikov, T. W. Hänsch, and I. Bloch, Nature 429, 277 (2004).

[10] L. D. Landau and E. M. Lifshitz, Statistical Physics (Butterworth-Heinemann, Oxford, 1998), 3rd ed.

[11] E. H. Hauge, Physica Nor. 4, 19 (1969); I. Fujiwara, D. ter Haar, and H. Wergeland, J. Stat. Phys. 2, 329 (1970); R. M. Ziff, G. E. Uhlenbeck, and M. Kac, Phys. Rep. 32, 169 (1977).

[12] H. D. Politzer, Phys. Rev. A 54, 5048 (1996); M. Gajda and K. Rzążewski, Phys. Rev. Lett. 78, 2686 (1997); P. Navez, D. Bitouk, M. Gajda, Z. Idziaszek, and K. Rzążewski, Phys. Rev. Lett. 79, 1789 (1997).

[13] M. Wilkens and C. Weiss, J. Mod. Opt. 44, 1801 (1997);
S. Grossmann and M. Holthaus, Phys. Rev. Lett. 79, 3557 (1997); N. L. Balazs and T. Bergeman, Phys. Rev. A 58, 2359 (1998).

[14] E. Buffet and J. V. Pulè, J. Math. Phys. 24, 1608 (1983).

[15] S. Giorgini, L. P. Pitaevskii, and S. Stringari, Phy. Rev. Lett. 80, 5040 (1998).

[16] V. V. Kocharovsky, V. V. Kocharovsky, and M. O. Scully, Phy. Rev. Lett. 84, 2306 (2000).

[17] S. Liu, H. Xiong, G. Huang, and Z. Xu, Phys. Rev. A 68, 065601 (2003).

[18] Z. Idziaszek, M. Gajda, P. Navez, M. Wilkens, and K. Rzążewski, Phys. Rev. Lett. 82, 4376 (1999).

[19] T. P. Meyrath, F. Schreck, J. L. Hanssen, C.-S. Chuu, and M. G. Raizen, Opt. Express 13, 2843 (2005).

[20] T. P. Meyrath, F. Schreck, J. L. Hanssen, C.-S. Chuu, and M. G. Raizen, Phys. Rev. A 71, 041604(R) (2005).

[21] Z. Hu and H. J. Kimble, Opt. Lett. 19, 1888 (1994); S. Kuhr, W. Alt, D. Schrader, M. Müller, V. Gomer, and D. Meschede, Science 293, 278 (2001).

[22] A MOT intensity of $8 \mathrm{~mW} / \mathrm{cm}^{2}$ and a detuning of about $5 \mathrm{MHz}$ are used for this measurement.

[23] For absorption imaging, the absolute accuracy in atom number is estimated to be $\pm 25 \%$.

[24] H. J. Metcalf and P. van der Straten, Laser Cooling and Trapping (Springer, New York, 1999).

[25] The rms technical noise is measured to be less than or equal to the following: $\Delta P_{x}=1 \%, \Delta P_{y}=5 \%$, $\Delta P_{z}=1 \%, \Delta l_{x}=0.5 \%$, and $\Delta l_{y}=0.2 \%$. Based on $\mathrm{TF}$ approximation in the three-dimensional regime with harmonic potentials, an estimate of the atom number fluctuation caused by technical noise gives $4.3 \%$. This limits the observation of sub-Poissonian fluctuations to $N<540$, as from $4.3 \%<N^{-1 / 2}$.

[26] A $99 \%$ confidence interval is defined as $\left[\sqrt{\frac{n-1}{\chi_{0.005}^{2}(n-1)}} \sigma_{N}, \sqrt{\frac{n-1}{\chi_{0.995}^{2}(n-1)}} \sigma_{N}\right]$, where $\sigma_{N}$ is the measurement standard deviation, $n$ is the number of samples, and $\chi_{0.005}^{2}(n-1), \chi_{0.995}^{2}(n-1)$ are the upper $0.5 \%$, $99.5 \%$ points of the chi-square distribution, respectively, with $n-1$ degrees of freedom. See, for example, R. V. Hogg and E. A. Tanis, Probability and Statistical 
Inference (Prentice Hall, New Jersey, 1997), 5th ed.

[27] A. M. Dudarev, M. G. Raizen, and Q. Niu, in preparation.

[28] J. Javanainen and M. Y. Ivanov, Phys. Rev. A 60, 2351 (1999).

[29] C.W. Gardiner, P. Zoller, R. J. Ballagh, and M. J. Davis, Phys. Rev. Lett. 79, 1793 (1997); C.W. Gardiner, M.D.
Lee, R. J. Ballagh, M. J. Davis, and P. Zoller, Phys. Rev. Lett. 81, 5266 (1998); M. D. Lee and C.W. Gardiner, Phys. Rev. A 62, 033606 (2000).

[30] L. D. Carr, M. J. Holland, and B. A. Malomed, J. Phys. B 38, 3217 (2005). 